\title{
Rapid electrochemical detection of vanillin in natural vanilla
}

\author{
Lifu Chen ${ }^{\dagger}$, Korbua Chaisiwamongkhol ${ }^{\dagger}$, Yuqi Chen ${ }^{\dagger}$, Richard G Compton* \\ Department of Chemistry, Physical and Theoretical Chemistry Laboratory, Oxford \\ University, South Parks Road, Oxford OX1 3QZ, UK
}

*To whom correspondence should be addressed

Email: richard.compton@chem.ox.ac.uk

Phone: +44 (0) $1865275957 \quad$ Fax: +44 (0) 1865275410

$\dagger$ : These authors contributed equally to the paper.

To be submitted to:

Electroanalysis 


\begin{abstract}
A commercially available and disposable multiwalled carbon nanotube screen-printed electrode (CNT-SPE) was employed to detect and determine vanillin compounds in natural vanilla. The voltammetric behaviour of vanillin at the CNT-SPE is examined and shown to be a sensitive method for quantifying vanillin. Linear calibration for vanillin in the range of 2.5-750 $\mu \mathrm{M}$ was obtained with a detection limit of $1.03 \mu \mathrm{M}$ and a quantification limit of $3.44 \mu \mathrm{M}$. The developed method comprises a simple sample preparation method and a sensitive electrochemical detection for the quantification of vanillin in vanilla pods and is an easy and simple procedure for manufacturers and consumers.
\end{abstract}

\title{
Key words:
}

Vanillin; Cyclic voltammetry; Screen-printed electrode; Quality control of vanilla products 


\section{Introduction}

Vanilla is one of the most widely used flavouring materials in confectionery, beverages, pharmaceuticals, food and perfumery around the world, ${ }^{[1]}$ and occupies a predominant market place in diverse foods among the variety of natural flavours in use today. The desirable flavour and aroma properties of vanilla are attributed to the presence of vanillin (4-hydroxy-3methoxybenzaldehyde), which is the main aromatic compound (1.0-2.0 wt $\%$ in cured vanilla pod ${ }^{[2]}$ ) among more than 200 components in natural vanilla. ${ }^{[3]}$

Natural Vanillin processes some biological properties that are beneficial for human health, including high antioxidant power ${ }^{[4]}$ as well as antimicrobial[5], anticarcinogenic[6] and antimutagenic[7] activities. It is thought to suppress chromosomal aberrations caused by UV and $\mathrm{X}$-rays, ${ }^{[8]}$ and also has been used as a DNA-PK inhibitor to prevent mutation at the CD59 locus on human chromosome[9] and to assist DNA strand repair[10]. However, the production of natural vanillin from vanilla covers only $0.2 \%$ of the market requirement and the remainder is synthetic vanillin made via chemical or biochemical processes.[11] Despite the fact that the synthetic vanillin is much cheaper and widely used in both food and non-food applications, extensive evidence indicates that it may cause headaches, nausea and vomiting, and even might be harmful to liver and kidney if excessive ingested.[12] Consequently, a simple, accurate and low-cost method for determination and quality control of vanillin is becoming more important in food science, medicine and pharmacology.

Hitherto, a variety of chemical techniques has been proposed and described for accurate determination of natural vanillin from various food samples or vanilla extracts including highperformance liquid chromatography (HPLC) ${ }^{[13]}$, gas chromatography (GC) ${ }^{[14]}$, thin layer chromatography (TLC) ${ }^{[15]}$, capillary electrophoresis (CE) ${ }^{[16], ~ U V ~ s p e c t r o p h o t o m e t r y ~}{ }^{[12,17]}$ and micellar electrokinetic chromatography[18]. However, these methods suffer from very high cost 
as well as difficult and time-consuming sample pretreatment processes, leading them inherently unattractive for use in detection of vanillin, particularly outside of a laboratory environment.

To address these problems, electroanalytical methods present an advantageous alternative for vanillin detection owing to their simplicity, rapid response and high sensitivity, and have been studied recently.[19] Many attempts have been made to modify the electrode surfaces to overcome the challenges of a relatively high overpotential of vanillin oxidation at bare electrode and poor reproducibility due to a fouling effect, leading to poor selectivity or/and sensitivity[20]. However, the performances reported suggest that a more straightforward approach would be highly favourable. In this paper, we report a simple and sensitive electroanalytical method for the determination of vanillin at a commercially available and disposable multiwalled carbon nanotube screen-printed electrode (CNT-SPE). More importantly, we demonstrate that this approach, as a proof-of-concept, can be used to detect and determine vanillin extracts from natural vanilla as a disposable and mass-producible sensing device, which is extremely simple, low-cost, accurate, reproducible and rapid. 


\section{Experimental}

\subsection{Chemicals and reagents}

All chemicals were of analytical grade and were used as received without further purification. Vanillin (99\% purity) was purchased from Alfa Aesar, UK. All solutions were made up with ultrapure water at resistivity not less than $18.2 \mathrm{M} \Omega \mathrm{cm}$ at 298K (Millipore, USA). Vanilla pods were purchased from a local supermarket, Tesco (Oxford, UK). The Britton-Robinson buffer, $0.05 \mathrm{M}$ at $\mathrm{pH} 1.8$, was prepared using boric acid (99.5\% purity, Aldrich), phosphoric acid (99.99\% purity, Aldrich) and acetic acid (99.5\% purity, BDH).

\subsection{Electrochemical measurements}

Electrochemical detections were performed using a $\mu$ Autolab III potentiostat (Metrohm-Autolab BV, Utrecht, Netherlands). The computer software, GPES v.4.9 (Metrohm Autolab B.V., NL) was used to control and measure all electrochemical experiments as well as to analyse results. Specifically, a linear baseline peak search function in GPES was used for peak analysis of solution phase results. For adsorption phase data, the currents were fitted to a $7^{\text {th }}$ order polynomial using Origin2017 software (Massachusetts, USA) to determine the true background current in the absence of vanillin. An illustration of baseline subtraction is shown in Figure S6. A three-electrode system in the form of a screen-printed electrode (SPE) was used throughout experimentation to fully show the capability of using a disposable unit for this method. The SPEs

(DropSens, Asturias, Spain) consist of a multiwalled carbon nanotube modified carbon working electrode (diameter of $4 \mathrm{~mm}$ ), a silver pseudo-reference electrode, and a carbon counter electrode.

\subsection{Real sample preparation}

For the authentic vanilla sample analysis, vanillin in natural vanilla pods was extracted by the following procedure. First, the vanilla pod was cut into pieces of a fixed length of $4 \mathrm{~mm}$. The 
samples were weighed in a small glass vial and the mass of each piece of sample was recorded. According to the weight of sample, ethanol was added into the vial as an extract solvent with the fixed sample mass to solvent amount ratio of $1 \mathrm{~g}$ of sample to $10 \mathrm{~mL}$ of ethanol. The vial was then sealed and subjected to sonication in the ultrasonic bath (FB15050, Fisher Scientific, 50/60 $\mathrm{Hz}, 80 \mathrm{~W}$, Germany) for 5-60 minutes to investigate the optimal sonication time.

For intra-pod study, 3 extract solutions were prepared from different parts of one pod. On the other hand, for inter-pod study, 3 extract solutions were prepared from three different pods following the same procedure as described above. The samples were sonicated for 30 minutes and extracted solutions were used for electrochemical measurements. To study the extraction method without ultrasonic-assisted the extractions were carried out using the same procedure with the vanilla pieces being soaked and left in ethanol for 6,10,20, and 30 minutes instead of sonication. Note that for maximum extraction study, vanilla pod was chopped into small pieces in order to increase surface area of sample. The mixture of sample and ethanol was placed in sonication bath with extraction times of half-day, 1 and 2 days.

\subsection{Analytical procedures}

A vanillin stock solution of $1.00 \mathrm{mM}$ was prepared with $0.05 \mathrm{M}$ Britton-Robinson buffer $\mathrm{pH} 1.8$ and the standard working solutions were then prepared by diluting the stock solution with a 0.05 M Britton-Robinson buffer.

For voltammetric characterisation of vanillin in the solution phase, $60 \mu \mathrm{L}$ of $100 \mu \mathrm{M}$ vanillin was dropped on the CNT-SPE so as to cover the three electrodes and followed immediately by five consecutive cyclic voltammetric scans recorded at a scan rate of $400 \mathrm{mVs}^{-1}$. In order to investigate the effect of scan rate on the voltammetric responses of vanillin, $60 \mu \mathrm{L}$ of $1.00 \mathrm{mM}$ vanillin was dropped on the CNT-SPE and cyclic voltammetry (CV) was performed and recorded as a function of scan rate $\left(25,50,100,200,300,400 \mathrm{mVs}^{-1}\right)$. 
For voltammetric characterisation of adsorbed vanillin, $60 \mu \mathrm{L}$ of $100 \mu \mathrm{M}$ vanillin was dropped on the CNT-SPE and left under open-circuit potential for 8 minutes to allow the adsorption of vanillin onto the electrode surface but little evaporation of the droplet. After this accumulation step, CVs were continuously performed for three scans. The effect of accumulation time was also investigated experimentally. $60 \mu \mathrm{L}$ of $100 \mu \mathrm{M}$ vanillin was dropped on the CNT-SPE and left under open circuit condition for different accumulation times in the range of 1-20 minutes. Cyclic voltammetric scans were recorded at a scan rate of $100 \mathrm{mV} \mathrm{s}^{-1}$.

For the analysis of the real samples, the extracts from vanilla pods were diluted with a $0.05 \mathrm{M}$ BR buffer solution pH 1.8 until the voltammetric response fell within the linear detection range. The real sample solutions were then dropped on CNT-SPEs and allowed to accumulate at opencircuit potential for 8 minutes prior to commencing voltammetric analysis at a scan rate of 100 $\mathrm{mV} \mathrm{s}^{-1}$. 


\section{Results and discussion}

This section first reports the solution phase voltammetric response of vanillin at CNT-screen printed electrodes measured using a high concentration of vanillin at the millimolar level. Second, the adsorption of vanillin onto the electrode surface is studied using dilute solutions and allowing the target to adsorb on the electrode surface. An appropriate waiting time for the adsorption is investigated and adopted. Finally, the extraction procedure of natural vanillin from vanilla pods is optimised, followed by the use of proposed electrochemical detection to quantify of vanillin content in the real samples.

\subsection{Voltammetric behaviour of vanillin at CNT-screen printed electrode}

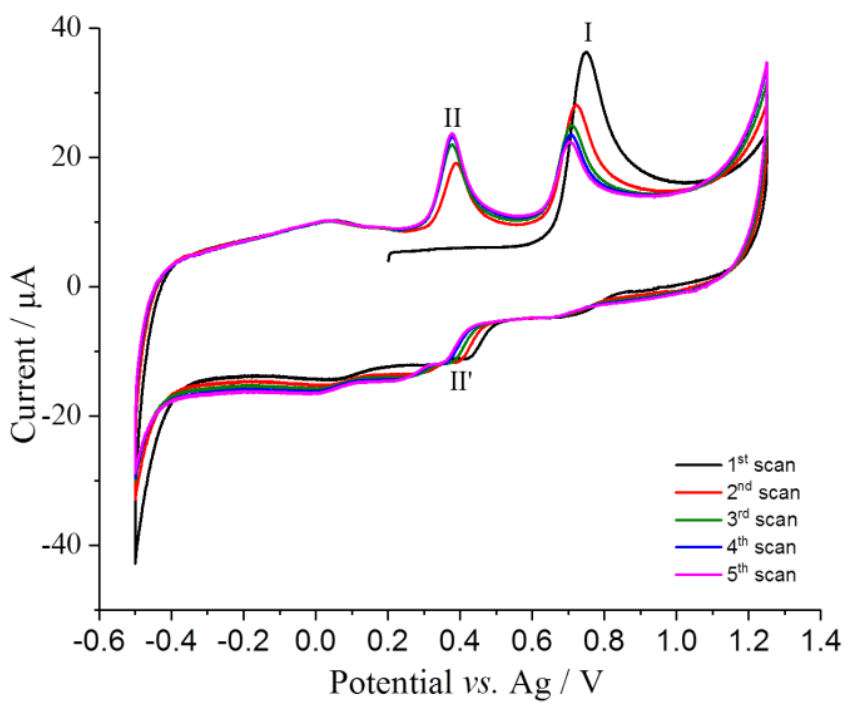

Fig. 1 The overlaid five consecutive scans of cyclic voltammetry of $100 \mu \mathrm{M}$ vanillin in a $0.05 \mathrm{M}$ BrittonRobinson buffer solution pH 1.8 at CNT-screen printed carbon electrode; scan rate $400 \mathrm{mV} \mathrm{s}^{-1}$.

The solution phase voltammetric behaviour and electroactivity of vanillin was first explored at CNT-screen printed carbon electrodes in pH 1.8 BR buffer. $60 \mu \mathrm{L}$ of $1 \mathrm{mM}$ vanillin in $0.05 \mathrm{M}$ BR buffer $\mathrm{pH} 1.8$ was dropped onto the CNT-screen printed carbon electrode and five consecutive scans of $\mathrm{CV}$ were recorded at $400 \mathrm{mV} \mathrm{s}^{-1}$. As shown in Fig. 1, a well-defined oxidative peak I was obtained at $+0.75 \mathrm{~V} v s$. Ag wire in the first scan from +0.2 to $+1.4 \mathrm{~V}$. The first oxidative peak is attributed to the oxidation of vanillin, as depicted in Scheme 1. A reduction peak appeared at 
$+0.42 \mathrm{~V}$ on the reverse scan, attributed to the reduction of the product from the chemically irreversible hydrolysis of the 2-methoxy group to form an $o$-benzoquinone moiety.[21] During the second scan, oxidative peak II wave was observed at $+0.39 \mathrm{~V}$ and formed a redox couple with reduction peak $\mathrm{II}^{\prime}$, corresponding to the reversible redox electrochemical process of $o$ benzoquinone and catechol substructures in vanillin.[22] Multi-scan CV recordings revealed that the oxidative peak I waves decreased upon the second and subsequent scans while oxidative peak II waves increased with the number of scan, indicating the intermediates are surface bound.

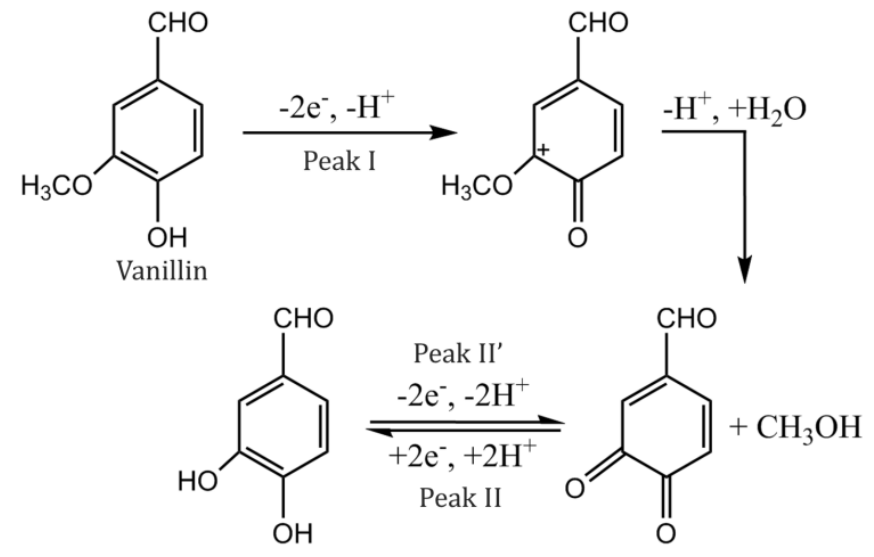

Scheme 1 Mechanism for the electrochemical reaction of vanillin 


\subsection{Effect of voltage scan rate}
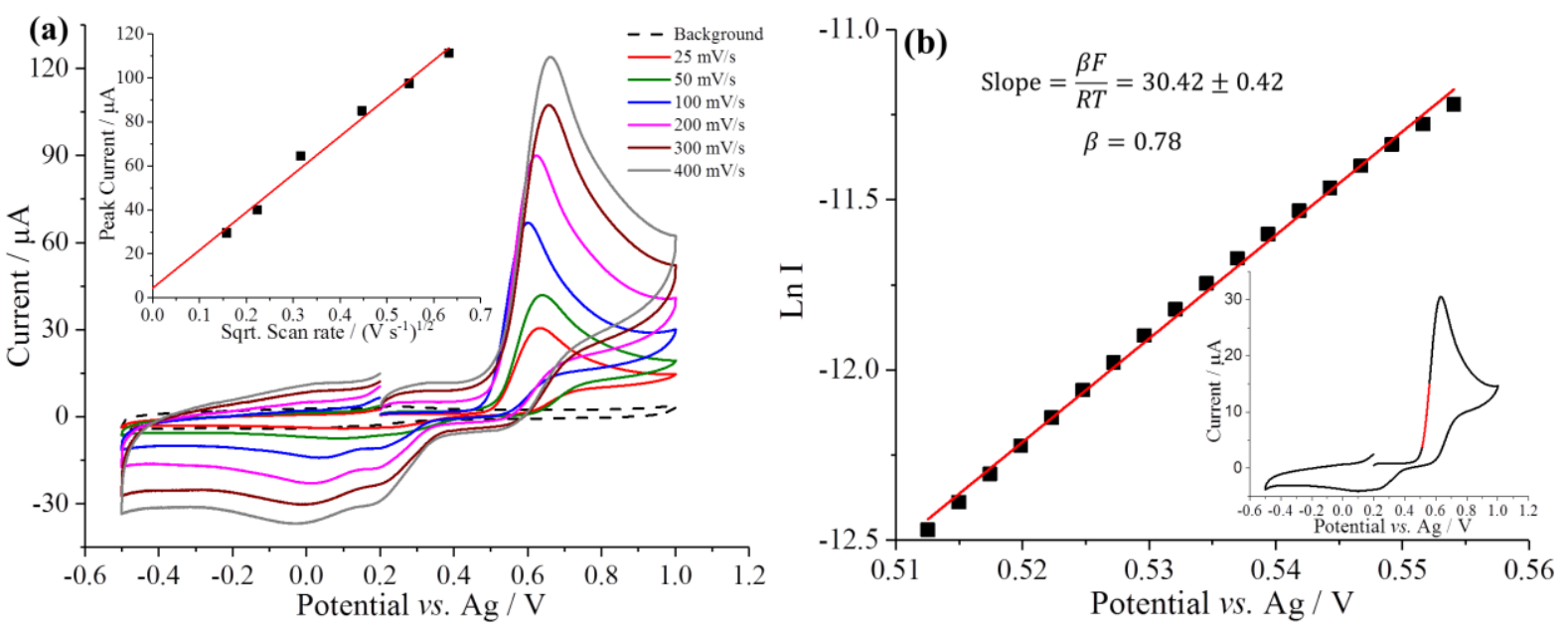

Fig. 2 (a) The first scans of cyclic voltammetry of $1 \mathrm{mM}$ vanillin in a $0.05 \mathrm{M}$ Britton-Robinson buffer solution pH 1.8 at CNT-screen printed carbon electrode at varying scan rates. Inset: the plot of peak current as a function of the square root of the scan rate from $25 \mathrm{mV} \mathrm{s}^{-1}$ to $400 \mathrm{mV} \mathrm{s}^{-1}$. (b) Tafel analysis for voltammogram of a CNT-screen printed carbon electrode in $1 \mathrm{mM}$ Vanillin at a scan rate of $25 \mathrm{mV} \mathrm{s}^{-1}$. The highlighted red region in inset voltammograms was selected as the Tafel analysis region, where the diffusion plays a near negligible role.[23]

The effect of scan rate on the solution phase oxidation peak currents of the first scans of the cyclic voltammetric responses of vanillin in $0.05 \mathrm{M}$ BR buffer pH 1.8 at CNT-screen printed carbon electrode was studied. Figure 2 shows the cyclic voltammograms of vanillin in the range of 25 to $400 \mathrm{mV} \mathrm{s}^{-1}$. It can be seen that the wave shape is almost constant and the peak currents increase with the increasing of scan rate from 25 to $400 \mathrm{mV} \mathrm{s}^{-1}$. The peak current was found to be directly proportional to the square root of scan rate, as depicted in Figure 2 Inset, suggesting that the oxidation of vanillin at high concentrations is under diffusion control of the electrode process. The apparent oxidative transfer coefficient $(\beta)^{[24]}$ was found to be 0.78 using Tafel analysis (Figure 2b), and the diffusion coefficient of vanillin in aqueous solution was estimated via the multiple electron-transfer, irreversible Randles-Sevcik equation[25] to be $6.7 \times 10^{-10}$ $\mathrm{m}^{2} / \mathrm{s}$.

\subsection{Voltammetric behaviour of adsorbed vanillin}



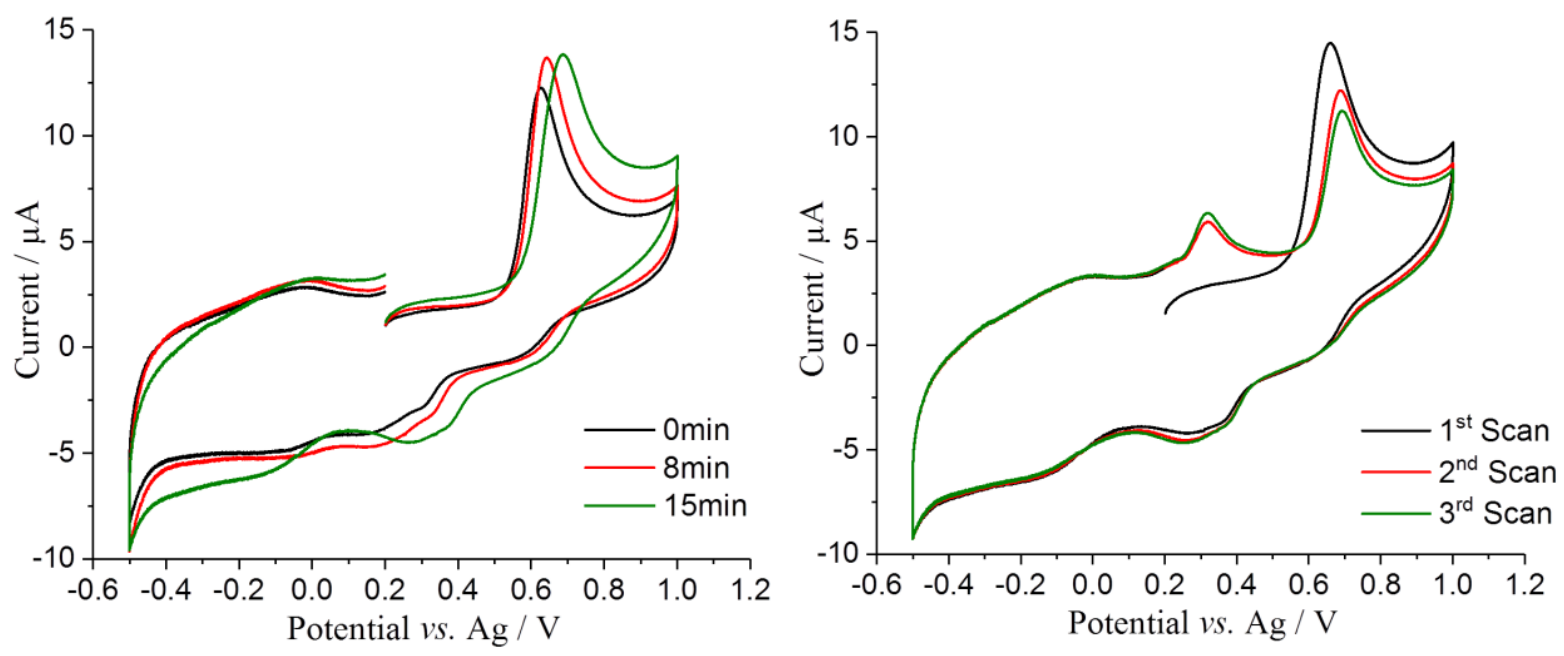

Fig. 3 (a) The first scans of cyclic voltammetry of $100 \mu \mathrm{M}$ vanillin in a $0.05 \mathrm{M}$ Britton-Robinson buffer solution pH 1.8 on CNT-screen printed carbon electrode; after open-circuit accumulation for 0 min (black), 8 min (red) and 15 min (green); scan rate $100 \mathrm{mV} \mathrm{s}^{-1}$. (b) The overlaid consecutive scans of cyclic voltammetry of adsorbed $100 \mu \mathrm{M}$ vanillin after open-circuit accumulation for 8 minutes; scan rate $100 \mathrm{mV} \mathrm{s}^{-1}$.

Having characterisation of electrochemical behaviour of vanillin in solution phase, voltammetric responses of adsorbed vanillin on electrode surface were considered next using low concentrations of vanillin where pre-concentration is necessary to obtain an analytically useful signal. $60 \mu \mathrm{L}$ of $100 \mu \mathrm{M}$ vanillin in a $0.05 \mathrm{M}$ Britton-Robinson buffer solution pH 1.8 was dropped onto CNT-screen printed carbon electrode and left for different time at open circuit potential before scanning. After the accumulation of vanillin, multi-scanning of CV was performed at scan rate of $100 \mathrm{mV} \mathrm{s}^{-1}$. As depicted in Figure 3a, the oxidation peak of vanillin becomes bigger with the longer accumulation time (from 0 to $8 \mathrm{~min}$ ) indicating the adsorption of vanillin onto electrode surface and this signal enhancement stabilises after 8 min showing the adsorption reach its dynamic equilibrium (maximum) at this point. This suggesting initially (at $0 \mathrm{~min}$ ) the peak is attributed to the oxidation of solution phase vanillin but after $8 \mathrm{~min}$ is a mixture of adsorbed and solution phase vanillin. A positive-shift of the oxidative peak potential at longer accumulation time is evident in Figure 3a, which can be explained that the increased amount of adsorbed molecules on the electrode surface at longer accumulation times which causes the peak to shift to slightly higher potentials as the oxidation is completed later in the 
scan. The consecutive voltammetric scans of adsorbed vanillin on CNT-SPE were also studied as shown in Figure $3 \mathrm{~b}$ and the obtained electrochemical respond is similar to solution phase vanillin. Specifically, the one oxidation peak of vanillin appeared during the first scan and decreased with the number of scans. A small reduction peak was obtained during the reverse scan and coupled with a new oxidation peak which appeared after the second scan.

\subsection{Effect of waiting time}
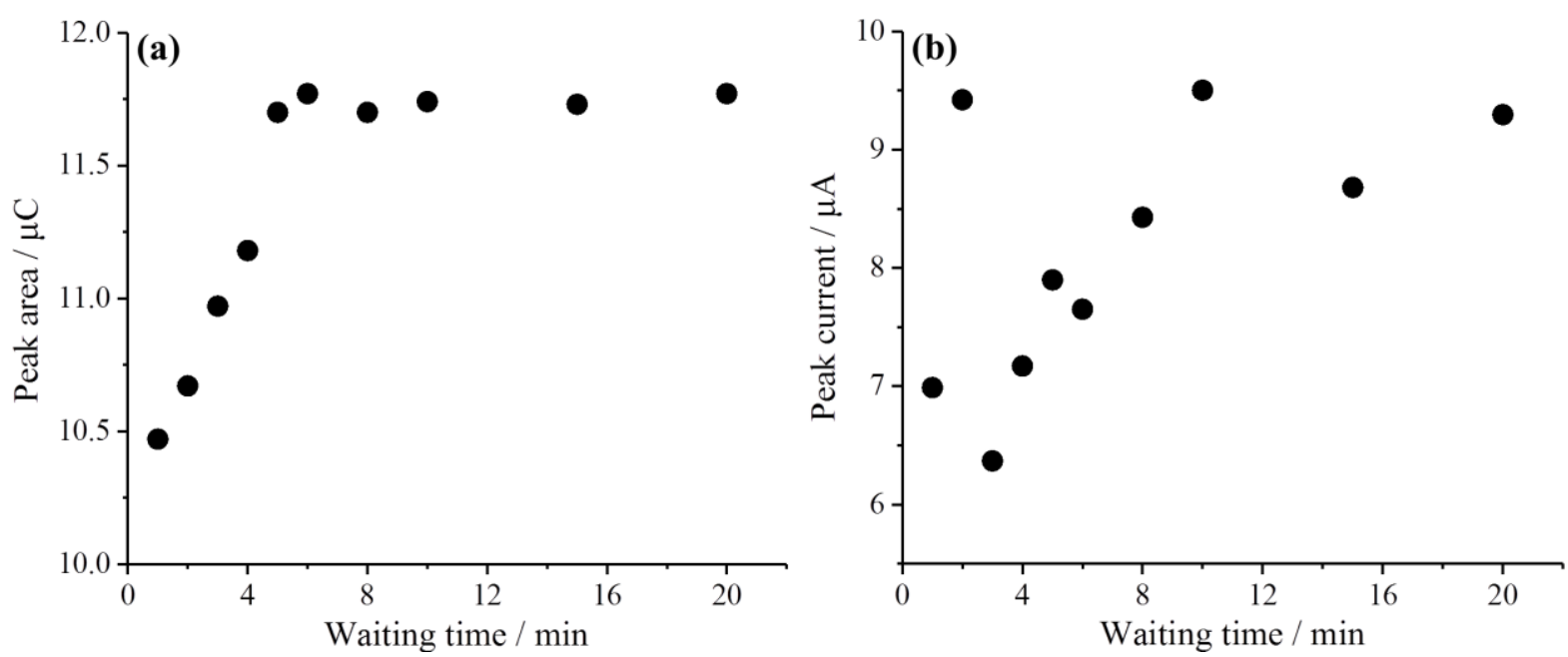

Fig. 4 The responses of oxidative peak area (a) and peak current (b) as a function of waiting time in $100 \mu \mathrm{M}$ of vanillin.

Having studied the voltammetric response of adsorbed vanillin, the effect of waiting time on the anodic peak responses of adsorbed vanillin at $\mathrm{pH} 1.8$ was studied at CNT-screen printed carbon electrodes under open circuit conditions. In order to optimise the waiting time, CVs of vanillin were performed in the range of $10-100 \mu \mathrm{M}$ at variable accumulation times. The vanillin solution of varying concentrations was dropped on CNT-SPE and left for waiting times ranging from 1 to 20 minutes at open circuit potential. After a certain waiting times, CV was performed and recorded from +0.2 to $+1.4 \mathrm{~V}$ as the first potential vortex and then reversing from $+1.4 \mathrm{~V}$ to -0.4 $\mathrm{V}$ as the second vortex to $+0.2 \mathrm{~V}$ as the end potential.

Figure 4 compares the peak area (charge) and peak current of oxidative peak as a function of adsorption time of vanillin compound on the electrode surface. Considering the result of $100 \mu \mathrm{M}$ 
vanillin, the peak charge increased significantly during the accumulation time from 1 to 5 minutes indicating an enhancement of vanillin uptake at the electrode surface and reached a plateau at $c a .8$ minutes, corresponding to maximum adsorption of vanillin at this concentration. However, the peak current plot is more scattered even though a similar trend could be seen. This is because the peak shape changes slightly after baseline correction and the peak potential shifts due to the silver pseudo-reference electrode. Therefore, peak charge is a more robust analytical parameter than peak current and is used in the further studies.

The maximum peak area of the plateau region is sensitive to the vanillin concentration (Figure S1 \& 4a), suggesting that the extent of maximum surface adsorption of vanillin onto the electrode is thermodynamically limited. An accumulation time for longer than 8 minutes gives the highest response towards the presence of vanillin. Therefore, an accumulation time of 8 minutes was chosen as the optimum time for further studies in respect of high sensitivity and short time of analysis.

\subsection{Analytical response of CNT-screen printed electrode}

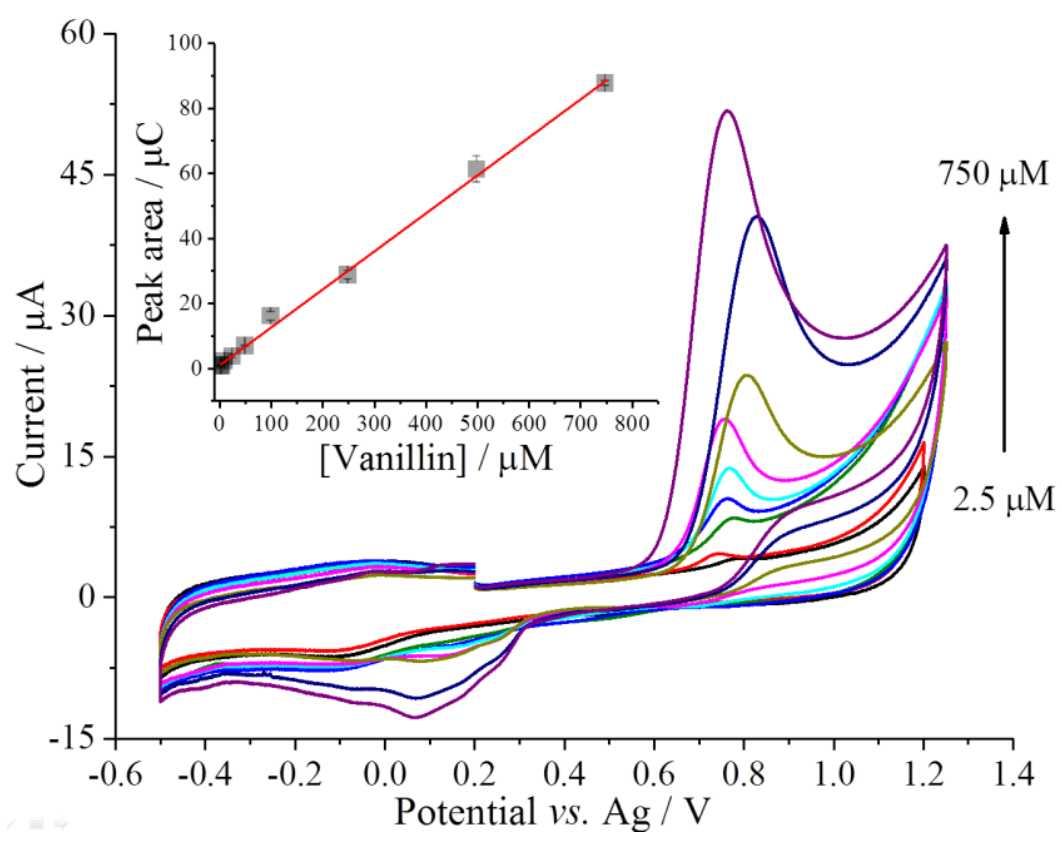

Fig. 5 Adsorptive stripping cyclic voltammetric responses of a CNT-screen printed electrode to increasing vanillin concentrations from 2.5 to $750 \mu \mathrm{M}$ in a $0.05 \mathrm{M}$ Britton-Robinson buffer solution pH 1.8, after open-circuit 
accumulation for 8 minutes; scan rate $100 \mathrm{mV} \mathrm{s}^{-1}$. Inset: the corresponding calibration curve plot using the oxidation peak.

Regarding the analytical performance of the CNT-SPE towards vanillin, cyclic voltammetry of varying concentrations of vanillin were tested. $60 \mu \mathrm{L}$ of vanillin standard solutions with concentrations in the range of 2.5-750 $\mu \mathrm{M}$ in a $0.05 \mathrm{M}$ Britton-Robinson buffer solution (pH 1.8) was dropped onto CNT-screen printed electrode. The experiments were conducted by first accumulating of vanillin compounds on CNT-SPE for 8 minutes under open-circuit condition. After adsorption of the analyte, CVs were recorded. Figure 5 depicts the cyclic voltammograms as a function of the concentration of vanillin. A linear relationship was established at CNT-SPE when peak area under oxidation peak was plotted with the concentrations of vanillin (inset Fig.5). The calibration curve is $Q / \mu C=(0.12 \pm 0.01)[$ vanillin $](\mu M)+(0.97 \pm 0.68 \mu C)$ in the range of 2.5-750 $\mu \mathrm{M}, \mathrm{N}=9, \mathrm{R}^{2}$ of 0.998 . The limit of detection (LOD) and limit of quantification values were calculated based on ten measurements of the low concentration of vanillin $(1 \mu \mathrm{M})$ to estimate the standard deviation $(\sigma)$. The limit of detection, determined by three times of the standard deviation $(3 \sigma)$, is $1.03 \mu \mathrm{M}$. The limit of quantification, estimated by ten times of the standard deviation $(10 \sigma)$ is $3.44 \mu \mathrm{M} .{ }^{[26]}$

\subsection{Analysis of a real sample (vanilla pod) on CNT-screen printed electrode}

Real samples of vanilla pod were analysed in order to evaluate the practicality of the herein developed method. To extract vanillin compound from vanilla pods, a sonication-assisted method was first used and effect of sonication time on vanillin extraction amount was studied. We investigated the extracted efficiency at different sonication times in the range of 6-60 minutes. A piece of the vanilla pod was cut from a long pod of vanilla and was weighed prior to addition of ethanol. Ethanol was added to vials contained samples with fixed ratio to the sample mass (1 g of sample to $10 \mathrm{~mL}$ ethanol). The samples were then subjected to sonication in an ultrasonic bath for the different time period. After sonication, the supernatant was collected and 
diluted with $0.05 \mathrm{M} \mathrm{BR}$ buffer (pH 1.8) until the voltammetric response fell within the linear detection range. $60 \mu \mathrm{L}$ of extract solutions was dropped onto CNT-screen printed electrode. The experiments were conducted by first accumulating vanillin compounds on CNT-SPE for 8 minutes under open-circuit condition and $\mathrm{CV}$ was performed at scan rate of $100 \mathrm{mV} \mathrm{s}^{-1}$. The analysed results are shown in Figure S2 and show that the extraction amount of vanillin increased with sonication time and reach the maximum at $c a .30 \mathrm{~min}$, where the results have been expressed as vanillin concentration in extracted solvent and then converted to vanillin content $(\% \mathrm{w} / \mathrm{w})$ of the original sample. Consequently, the extraction procedure with sonication time of 30 minutes was used for the further extractions of a real sample.
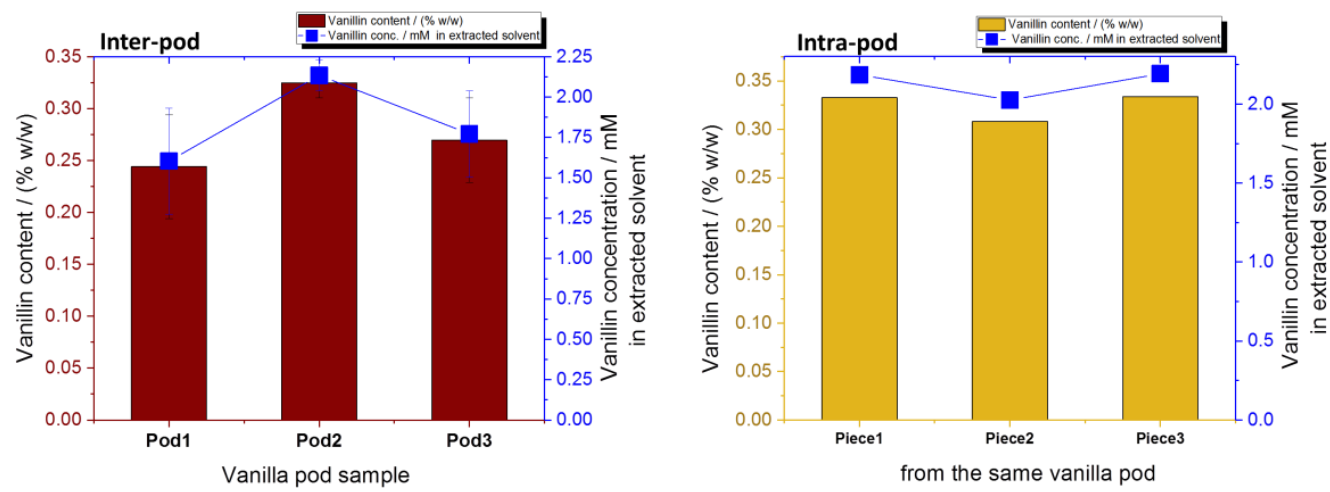

Fig. 6 Plots of vanillin content $(\% \mathrm{w} / \mathrm{w})$ and vanillin concentration in extracted solvent obtained from vanilla pod for inter-pod (A) and intra-pod (B) study.

Having investigated the sonication time intra- and inter-pods variability was studied to verify this method. Sample preparation procedure was described in section 2.3. Briefly, vanilla pods were cut into small pieces with the length of $4 \mathrm{~mm}$ and were weighed. Ethanol was added to vials contained samples with fixed ratio to the sample mass ( $1 \mathrm{~g}$ of sample to $10 \mathrm{~mL}$ ethanol). The vials were then sonicated for 30 minutes. The supernatant was collected and diluted with 0.05 M BR buffer solution at pH 1.8 until the voltammetric response fell within the linear detection range. In terms of intra-pods study, three extracts of different parts of the same pod 
were prepared followed the method mentioned above. For inter-pods study, $4 \mathrm{~mm}$ of vanilla pods were sampling from three different pods. Following the similar sample preparation, the voltammetric procedure was carried out and quantifications were performed by means of calibration curve method. Figure 6 illustrates analysed result from inter- and intra-pods study suggesting that there is only small variation between samples. Specifically vanilla content from 3 different vanilla pods (the same brand but different batches), inter-pod study, was found to be $0.24 \pm 0.05 \% \mathrm{w} / \mathrm{w}, 0.32 \pm 0.01 \% \mathrm{w} / \mathrm{w}$, and $0.27 \pm 0.04 \% \mathrm{w} / \mathrm{w}$ for vanilla pod no.1, no.2, and no.3 respectively. In terms of intra-pod study, vanilla content was found to be $0.32 \pm 0.01 \% \mathrm{w} / \mathrm{w}$ $(n=3)$

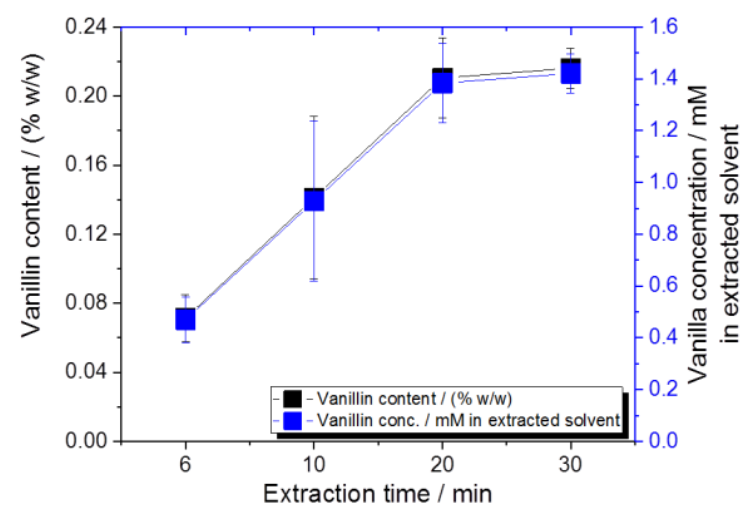

Fig. 7 Plot of vanillin content $(\% \mathrm{w} / \mathrm{w})$ and vanillin concentration in extracted solvent at a different extraction time without sonication.

Having evidenced the usability of the proposed method to quantify vanillin content in real samples, the extraction method was simplified to facilitate the developed method for on-site analysis. Extraction procedures without sonication were investigated. Pieces of $4 \mathrm{~mm}$ of pod were soaked in the ethanol for $6,10,20$, and 30 minutes respectively. The voltammetric procedure was carried out followed the procedure mentioned above and quantifications were also performed using calibration curve method. Considering vanillin content obtained from sample extracted without sonication, the results show that the vanilla contents increase with 
extraction time and reach a plateau at 20 minutes of extraction time as can be seen in Figure 7. This vanillin content without the aid of sonication was found to be $0.21 \pm 0.03 \% \mathrm{w} / \mathrm{w}$ which is slightly lower than that from extraction with sonication $(0.32 \pm 0.01 \% \mathrm{w} / \mathrm{w})$. This result suggested that ultrasonic-assisted extraction slightly improves the extraction of vanillin from a vanilla pod. However, in terms of facilitating the practical use outside a laboratory, the extraction method without sonication may be used as a simplified extraction procedure to facilitate comparative measurements between samples. From this result, we propose that we can simplify the extraction method by simply soaking the sample with ethanol for 20 minutes. Note that value reported is based on the simplified extraction method where the extraction efficiency is not $100 \%$ (Figure S3). From the quantification result, we reported that the amount of vanillin in vanilla pod sample based on the simplified extraction method of 20 minutes extraction was $0.21 \pm 0.03 \% \mathrm{w} / \mathrm{w}(\mathrm{n}=3)$.

The developed method was further applied on unmodified carbon screen printed electrode (carbon-SPE) to prove the capability of the method when employing a much cheaper electrode. Following the similar procedure, the calibration curve was first constructed for studying analytical performance of carbon-SPE and for using to determination of vanillin content in real samples. A linear relationship of vanillin concentrations and peak area was obtained in the range of 5-300 $\mu \mathrm{M}$ as shown in Figure S4 in SI. For real sample analysis, an analogous experiment of variable extraction time without sonication was performed at 6, 10 and 20 minutes extraction time. The similar trend of vanillin content plotted against extraction time was obtained (see Figure S5). By employing the simplified extraction method of 20 minutes extraction, the amount of vanillin in vanilla pod sample was found to be $0.28 \pm 0.03 \% \mathrm{w} / \mathrm{w}(\mathrm{n}=3)$. The vanillin content obtained from the use of CNT-SPE and carbon-SPE are likely comparable suggesting that the developed method can be applied to use with both electrodes. Utilising the CNT-SPE enables a larger linear range and a lower limit of detection whilst the C-SPE is much cheaper. 


\section{Conclusions}

A CNT-CPE has been successfully utilised for electrochemically determination of vanillin in standard solutions and in a variety of real vanilla samples. The detection limit of $1.03 \mu \mathrm{M}$ and quantification limit of $3.44 \mu \mathrm{M}$ were obtained. The extraction of vanillin from real vanilla pods can be simply achieved via leaving fixed length pieces of pod in ethanol for a certain time. This study contributes to the establishment of simple extraction methods and electrochemical detection for rapid and sensitive vanillin quantification in vanilla pods and for routine purpose analysis. The method also has been proved to be applicable when employing unmodified carbon screen printed electrode which offers similar advantages to CNT modified screen printed electrode but much more cost effective. This method can be easily applied as a mass-produced, disposable sensor by manufacturers and consumers.

\section{Acknowledgement}

K.C. thanks the Royal Thai Government for financially supporting her DPhil. 


\section{References}

[1] a) A. S. Ranadive, Developments in food science. 1994, 34, 517-577; b) H. B. Heath, Flavor technology, profiles, products, applications, Avi Pub. Co., 1978.; c) Y. H. Hui, Encyclopedia of food science and technology, Wiley, 1992.

[2] A. Sharma, S. C. Verma, N. Saxena, N. Chadda, N. P. Singh and A. K. Sinha, Journal of separation science 2006, 29, 613-619.

[3] E. Anklam, S. Gaglione and A. Müller, Food chemistry 1997, 60, 43-51.

[4] a) T. Sawa, M. Nakao, T. Akaike, K. Ono and H. Maeda, Journal of Agricultural Food Chemistry 1999, 47, 397-402; b) P. Teissedre and A. Waterhouse, Journal of Agricultural Food Chemistry 2000, 48, 3801-3805.

[5] C. Boonchird and T. Flegel, Canadian Journal of Microbiology 1982, 28, 1235-1241.

[6] K. Akagi, M. Hirose, T. Hoshiya, Y. Mizoguchi, N. Ito and T. Shirai, Cancer letters 1995, 94, 113-121.

[7] L. R. Ferguson, Mutation Research/Fundamental Molecular Mechanisms of Mutagenesis 1994, 307, 395-410.

[8] Y. Sasaki, T. Ohta, H. Imanishi, M. Watanabe, K. Matsumoto, T. Kato and Y. Shirasu, Mutation Research Letters 1990, 243, 299-302.

[9] D. L. Gustafson, H. R. Franz, A. M. Ueno, C. J. Smith, D. J. Doolittle and C. A. Waldren, Mutagenesis 2000, 15, 207-213.

[10] S. Durant and P. Karran, Nucleic acids research 2003, 31, 5501-5512.

[11] N. J. Walton, M. J. Mayer and A. Narbad, Phytochemistry 2003, 63, 505-515.

[12] Y. Ni, G. Zhang and S. Kokot, Food chemistry 2005, 89, 465-473.

[13] a) L. S. de Jager, G. A. Perfetti and G. W. Diachenko, Journal of chromatography A 2007, 1145, 83-88; b) K. N. Waliszewski, V. T. Pardio and S. L. Ovando, Food chemistry 2007, 101, 1059-1062; c) G. Lamprecht, F. Pichlmayer and E. R. Schmid, Journal of Agricultural Food Chemistry 1994, 42, 1722-1727.

[14] a) A. Pérez-Silva, E. Odoux, P. Brat, F. Ribeyre, G. Rodriguez-Jimenes, V. Robles-Olvera, M. García-Alvarado and Z. Günata, Food chemistry 2006, 99, 728-735; b) T. Sostaric, M. C. Boyce and E. E. Spickett, Journal of Agricultural Food Chemistry 2000, 48, 5802-5807.

[15] A. Gerasimov, N. Gornova and N. Rudometova, Journal of Analytical Chemistry 2003, 58, 677-684.

[16] a) A. Panossian, G. Mamikonyan, M. Torosyan, E. Gabrielyan and S. Mkhitaryan, Analytical chemistry 2001, 73, 4379-4383; b) M. Ohashi, H. Omae, M. Hashida, Y. Sowa and S. Imai, Journal of Chromatography A 2007, 1138, 262-267.

[17] A. Longares-Patrón and M. Canizares-Macias, Talanta 2006, 69, 882-887.

[18] M. C. Boyce, P. R. Haddad and T. Sostaric, Analytica chimica acta 2003, 485, 179-186.

[19] a) D. Zheng, C. Hu, T. Gan, X. Dang and S. Hu, Sensors Actuators B: Chemical 2010, 148, $247-$

252; b) Y. Yardım, M. Gülcan and Z. Şentürk, Food chemistry 2013, 141, 1821-1827; c) L. Shang,

F. Zhao and B. Zeng, Food chemistry 2014, 151, 53-57; d) P. Deng, Z. Xu, R. Zeng and C. Ding, Food chemistry 2015, 180, 156-163.

[20] J. L. Hardcastle, C. J. Paterson and R. G. Compton, Electroanalysis 2001, 13, 899-905.

[21] L. Papouchado, J. Bacon and R. Adams, Journal of Electroanalytical Chemistry Interfacial Electrochemistry 1970, 24, A1-A5.

[22] a) A. Nilsson, U. Palmquist, T. Pettersson and A. Ronlán, Journal of the Chemical Society, Perkin Transactions 1 1978, 696-707; b) D. Nematollahi, H. Goodarzi and E. Tammari, Journal of the Chemical Society, Perkin Transactions 2 2002, 829-834; c) D. Nematollahi, M. Alimoradi and S. W. Husain, Electroanalysis 2004, 16, 1359-1365.

[23] D. Li, C. Lin, C. Batchelor-McAuley, L. Chen and R. G. Compton, Journal of Electroanalytical Chemistry 2018, 826, 117-124. 
[24] a) R. Guidelli, R. G. Compton, J. M. Feliu, E. Gileadi, J. Lipkowski, W. Schmickler and S. Trasatti, Pure Applied Chemistry 2014, 86, 245-258; b) R. Guidelli, R. G. Compton, J. M. Feliu, E. Gileadi, J. Lipkowski, W. Schmickler and S. Trasatti, Pure Applied Chemistry 2014, 86, 259-262.

[25] R. G. Compton and C. E. Banks, Understanding voltammetry, World Scientific, 2018.

[26] D. A. Skoog, D. M. West and F. J. Holler, Fundamentals of Analytical Chemistry, Brooks Cole Publishing, 2004. 